Document downloaded from:

http://hdl.handle.net/10251/61863

This paper must be cited as:

Navarro, MD.; Lloréns Rodríguez, R.; Noé, E.; Ferri, J.; Alcañiz Raya, ML. (2013). Validation of a low-cost virtual reality system for training street-crossing. A comparative study in healthy, neglected and non-neglected stroke individuals. Neuropsychological Rehabilitation. 23(4):597-618. doi:10.1080/09602011.2013.806269.

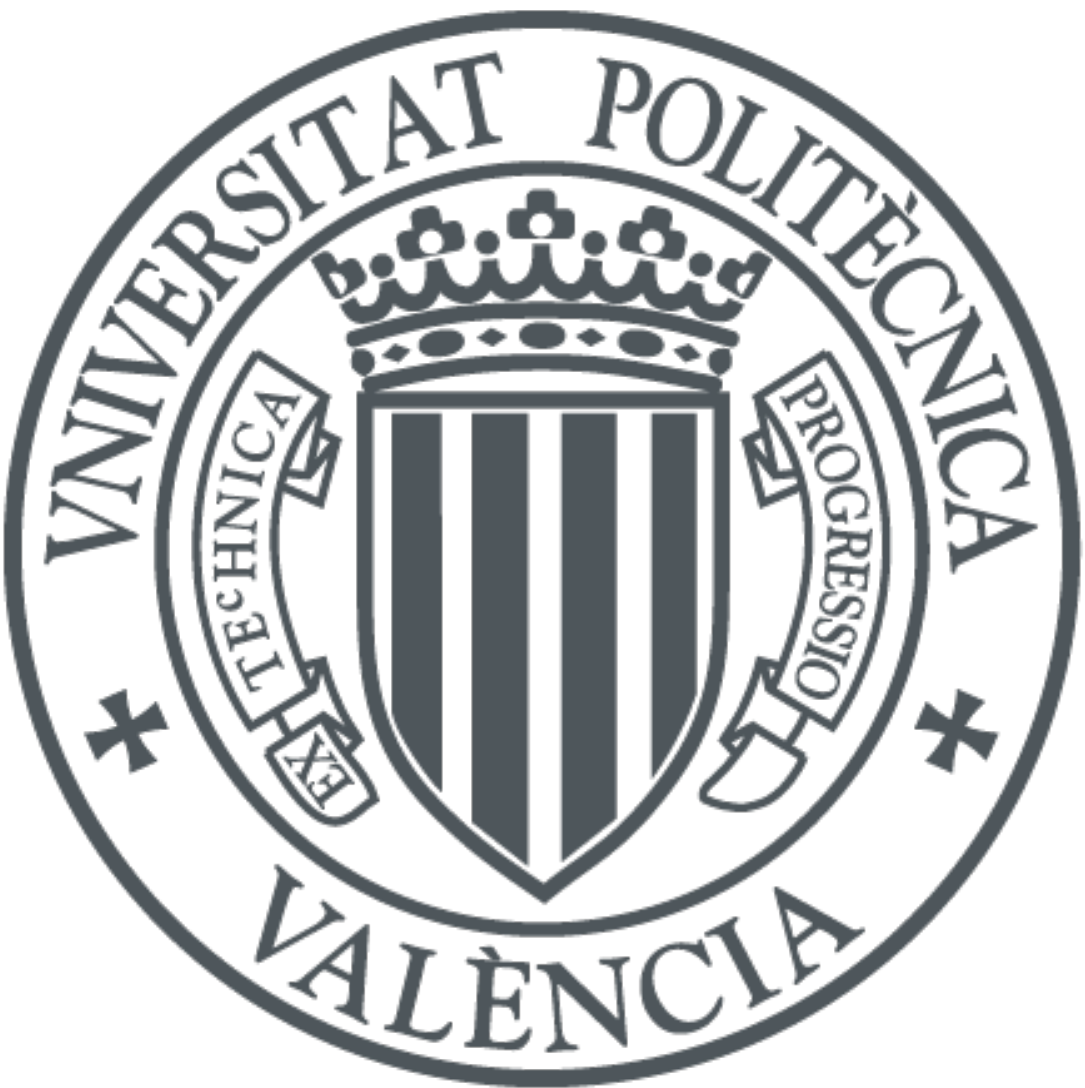

The final publication is available at

http://dx.doi.org/10.1080/09602011.2013.806269

Copyright Taylor \& Francis (Routledge): STM, Behavioural Science and Public Health Titles

Additional Information 
Running head: VALIDATION OF A VIRTUAL STREET-CROSSING SYSTEM

Validation of a low-cost virtual reality system for training street-crossing. A comparative study in healthy, neglected and non-neglected stroke individuals.

María-Dolores Navarro ${ }^{1}$, Roberto Lloréns ${ }^{2}$, Enrique Noé ${ }^{1}, J^{\prime}$ an Ferri ${ }^{1}$, Mariano Alcañiz ${ }^{2,3}$

${ }^{1}$ Servicio de Neurorrehabilitación de los Hospitales NISA. Fundación Hospitales NISA, c/ Río Tajo

$$
\text { 1, 46011, Valencia, Spain. }
$$

${ }^{2}$ Instituto Interuniversitario de Investigación en Bioingeniería y Tecnología Orientada al Ser Humano, Universitat Politècnica de València, Camino de Vera s/n, 46022 Valencia, Spain.

${ }^{3}$ Ciber, Fisiopatología Obesidad y Nutrición, CB06/03 Instituto de Salud Carlos III, Av. Sos Baynat s/n, Univesity of Jaume I, 12071 Castellón, Spain 


\begin{abstract}
Unilateral spatial neglect is a common consequence of stroke that directly affects the performance of activities of daily living. This impairment is traditionally assessed with paper-and-pencil tests that can lack correspondence to real life and are easily compensated. Virtual reality can immerse patients in more ecological scenarios, thus providing therapists with new tools to assess and train the effects of this impairment in simulated real tasks. This paper presents the clinical validation and convergent validity of a low-cost virtual reality system for training street-crossing in stroke patients with and without neglect. The performance of neglected patients was significantly worse than the performance of non-neglected and healthy participants. In addition, several correlations between the scores in the system and in the traditional scales were detected.
\end{abstract}


Acknowledgments

The authors wish to thank the staff and patients of the Hospital NISA Valencia al Mar for their tireless and collaborative attitude, and José Antonio Lozano and José Antonio Gil for their early work. The authors also wish to thank the staff of LabHuman, specially José Roda, for their technical support.

This study was funded in part by Ministerio de Educación y Ciencia Spain, Projects Consolider-C (SEJ2006-14301/PSIC), “CIBER of Physiopathology of Obesity and Nutrition, an initiative of ISCIII" and the Excellence Research Program PROMETEO (Generalitat Valenciana. Conselleria de Educación, 2008-157). 


\section{Validation of a Low-Cost Virtual Reality System for Training Street-Crossing. A Comparative Study in Healthy, Neglected and Non-Neglected Stroke Individuals}

\section{Introduction}

Unilateral spatial neglect (USN) is a common and heterogeneous clinical consequence usually observed after a damage to the non-dominant cerebral hemisphere. USN affects the perception of contralesional environmental stimuli and can manifest in the visual, auditory, and tactile channels (Buxbaum et al., 2004). Clinically, USN can impair motor, visual, and sensory perceptions and can be caused by a number of widespread lesions affecting cortical and subcortical areas, particularly in the right hemisphere (Allegri, 2000). USN can manifest in personal space, affecting activities such as dressing and hair combing; in peripersonal space, affecting activities such as eating and working; and in extrapersonal space, affecting activities such as walking and driving. Different combinations of symptoms, lesions, and deficits can occur in different patients at different times. This makes assessment a difficult task and also explains why even though many treatments have been tried, none has been found to be uniformly successful or consistently effective. A recent evidence-based review of rehabilitation therapies for USN concluded that the effectiveness of rehabilitation for neglect remains unproven (Bowen \& Lincoln, 2007). This is particularly relevant in the chronic stage of USN, which has traditionally been associated with poor functional recovery (Appelros, Karlsson, Seiger, \& Nydevik, 2002; Jehkonen, Laihosalo, \& Kettunen, 2006).

The severity of this syndrome has traditionally been assessed with paper-and-pencil tests, including line crossing, line bisection, letter and star cancellation, and copy and drawing tasks (Allegri, 2000). However, most of these measures lack correspondence to activities of daily living (ADL). Moreover, visual scanning training can lead patients to learn how to compensate on these 
tasks. Thus, it is common for patients to improve their scores on paper-and-pencil tests through practice, without showing an associated improvement in real-life situations (Bowen \& Lincoln, 2007). To overcome these limitations new behavioral psychometric tests have recently been developed. For instance, the Rivermead Behavioural Inattention Test (BIT) assesses everyday skills, such as phone dialing, coin sorting, and map navigation (Wilson, Cockburn, \& Halligan, 1987). Over the past few years, the BIT has become one of the most widely used test for assessing USN. However, the long duration of this test is time-consuming for clinical staff and can be tiresome for many patients, particularly those with prominent attentional problems. The need for a rehabilitative therapist to administer the test and its psycholinguistic requirements may also prevent the widespread use of the BIT.

The introduction of virtual reality (VR) to the field of neurorehabilitation has given rise to new tools for the assessment and rehabilitation of cognitive impairments that were designed to overcome the limitations of traditional tests and interventions (Rose, Brooks, \& Rizzo, 2005). VR systems can recreate safe, ecological, and individualized 3D environments where patients are required to perform specific actions to achieve a goal. As an assessment tool, VR can register and objectively measure the performance of patients within the virtual world and their behavioral responses. There is a growing body of research focused on the use of VR in the assessment and rehabilitation of USN (Table 1).

(Table 1 about here)

VR allows for intensive, repetitive, and motivational training, factors clearly related to the speed of the learning processes (Krakauer, 2006; Rizzo et al., 2004). In addition, VR allows for task-specific training in ecological environments (Krakauer, 2006; Rizzo \& Buckwalter, 1997; Rizzo et al., 2004), which increases the generalizability and transferability of learning to the real 
world through more efficient plasticity mechanisms. VR also allows for the recreation of potentially hazardous real-life situations, such as street crossing, which cannot otherwise be trained. Street crossing involves intact attentional, perceptual and executive skills to evaluate whether traffic conditions guarantee the safe performance of the task. Virtual street-crossing systems are particularly interesting because they provide therapists with ecological and behavioral data for the evaluation of extrapersonal neglect and patient autonomy in the outdoor environment. Virtual street-crossing systems have also been used to educate children (McComas, MacKay, \& Pivik, 2002; Schwebel \& McClure, 2010; Thomson, Tolmie, Foot, Whelan, Sarvary, \& Morrison, 2005), to study the performance of pedestrians (Simpson, Johnston, \& Richardson, 2003; Wu, Ashmead, \& Bodenheimer, 2009), and to rehabilitate gait in stroke patients (Boian, Burdea, Deutsch, \& Winter, 2004).

Three previous studies have used 3D virtual street environments to assess (Kim et al., 2010; Peskine et al., 2011) and treat (Katz, Ring, Naveh, Kizony, Feintuch, \& Weiss, 2005; Weiss, Naveh, \& Katz, 2003) USN in a stroke population. While the assessment systems allowed subjects to interact using a head mounted display (HMD), a head tracker, and a mouse, the training system used a desktop monitor and a keyboard. HMDs can provide higher levels of immersion, but their cost, availability, and discomfort can make their use in neurorehabilitation services difficult. Though the three systems recreated a virtual street environment, only the training system required the participants to cross a street. Moreover, the virtual environment was unrealistic. No previous study has examined the correlation between VR outcomes and USN tests (or other neuropsychological tests).

The objective of this work was to design and validate a low-cost virtual street-crossing system that could be easily integrated in the clinical setting for training this task within the safety of the hospital facility. Since neglect was one of our primary therapeutic goals, we examined the convergent validity of our tool by analyzing the correlation of the VR outcomes with other 
traditional attentional measures. Finally, since stroke patients could have some difficulties to interact with VR technology we wanted to explore the usability of the system.

\section{Method}

\section{Participants}

A total of 139 acquired brain injury patients who were attending a neurorehabilitation program in a large metropolitan hospital were considered as potential candidates for the present study. The inclusion criteria were: 1) ischemic or hemorrhagic stroke; 2) age $>35$ and $<70 ; 3$ ) fairly good cognitive condition (Mini-Mental State Examination (MMSE) (Folstein, Folstein, \& McHugh, 1975) cut-off $\geq 23$ ). Exclusion criteria were: 1) patients with severe dementia or aphasia (Mississippi Aphasia Screening Test (MAST) (Romero, Sanchez, Marin, Navarro, Ferri, \& Noe, 2012) < 45); 2) patients whose visual or hearing impairment does not allow possibility of interaction with the system; 3) patients with sensorimotor alterations that can interfere with the performance with the system. After applying the inclusion and exclusion criteria, 32 stroke patients (20 men and 12 women) were enrolled in the study. These subjects were $54.8 \pm 12.2$ years old (mean \pm standard deviation), with a chronicity of $397.8 \pm 241.9$ days, and had $11.8 \pm 4.3$ years of education. 19 patients suffered an ischemic stroke and 13 patients suffered an intracerebral hemorrhage. 7 patients had a left-sided lesion and 25 patients had a right-sided lesion. Although most patients with right-sided lesions showed some degree of USN upon clinical examination, the operational criteria for the diagnosis of USN in this study was defined as a score below 129 on the conventional subtests of the BIT (Hartman-Maeir \& Katz, 1995). According to this criterion, the USN group consisted of 17 patients, while the non-USN group consisted of 15 patients (Table 2). The BIT scores of the USN and the non-USN group were $99.59 \pm 20.09$ and $140.93 \pm 11.93$, respectively. There was a tendency towards greater chronicity in stroke patients without USN 
compared with those with USN $(p=.06)$ and a clear predominance of USN in those patients with a right hemispheric stroke. Time under treatment was significantly higher in patients without USN compared with patients with USN $(p<.001)$.

In addition to the stroke patients, 15 healthy subjects who satisfied the established criteria of age and cognitive condition were recruited to form a control group. The control sample consisted of 12 men and 3 women, $54.7 \pm 5.7$ years old, and $11.3 \pm 2.7$ years of education. No significant differences in age, sex or years of education were found when comparing the control subjects with stroke patients with and without USN (Table 2).

(Table 2 about here)

\section{Materials}

Hardware. The VR street-crossing system consists of a standard PC, an audio-visual output system, a TrackIR 4:PRO (NaturalPoint, 2012) infrared tracking system, and a joystick. The output system consists of a video display (for instance, an LCD screen or projector) and an audio system. The system enables positional audio, allowing for different speaker configurations. The tracking system estimates the real-world position of the participant's head with 6 degrees of freedom. The tracking system consists of an infrared camera, which was attached to the upper side of the screen, and a clip with a constellation of three reflective marks, which was mounted on a cap. Only the yaw angle is transferred to the virtual world to represent the rotation of the head when exploring the traffic conditions of the roads. Navigation within the virtual environment (VE) is enabled by the joystick. The system allows subjects to walk (forward, backward, left, and right), stop, and turn.

For the clinical validation, the virtual street-crossing system run on an Intel® Core ${ }^{\mathrm{TM}} 2$ T5500@1.66GHz with 1 GB of RAM and a NVIDIA® GeForce® Go 7600 MB video card with Windows XP. A 47" LCD screen and a 5.1 sound system were used to provide audiovisual 
feedback.

Software. The virtual system recreates a real street intersection with a central roundabout in the city of Valencia (Spain). The interactive area of the VE consists of a crosswalk that intersects 2 two-way roads with median strips that lead to the roundabout. The virtual world is presented using a first-person view (Figure 1). A random number of cars drive through the roads and behave in accordance with the traffic conditions. As the cars approach the participant's position, they brake, which is also indicated by the sound of screeching brakes. If the cars pass near the participant's position, a horn sound is played. If the cars enter the participant's space, an accident is represented through a sudden camera movement to the ground and a text message.

(Figure 1 about here)

Within the VE, participants can walk down a restricted area of the street and look both ways to check the traffic conditions. Participants are able to move freely in the virtual world using a joystick. The displacements of the stick are transferred to the VE using a strafing technique, which allows the participants to move forwards and sideways. It is also possible to turn around by pressing a joystick button. However, the heading of the VE is estimated from the yaw angle of the head. The yaw angle is amplified in such a way that its maximum value, defined when the participants are facing the edge of the screen, represents $90^{\circ}$. Specifically, when the real yaw angle is less than $10^{\circ}$, the yaw is multiplied by 1.5 . When the yaw angle is greater than $10^{\circ}$, the yaw is multiplied by 2 .

The conditions of the virtual environment are fully customizable. Therapists can add obstacles, such as traffic lights, litter baskets and fences that the participants must dodge; control auditory cues to guide participants in the virtual task; and launch left- or right-sided distractors, such as ambulance lights and sounds. 
The street-crossing system provides two different play modes, a free mode that allows participants to move freely within the virtual environment without restrictions and a session mode that allows participants to move from an initial point to an end point (a large department store) and then return. Correct performance in the session mode includes twice checking the direction from which cars come, which entails turning the head to that side twice, and afterward safely crossing the street. Safe street crossing entails estimating a traffic gap that ensures the participant will reach the opposite sidewalk while avoiding collisions with cars and other dangerous situations. All session data are recorded by the system.

\section{Procedure}

The study took place in a quiet room in a specialized neurorehabilitation unit in a large metropolitan hospital. The environment was controlled to try to avoid all potential attentional distractors. Participants were seated $1.5 \mathrm{~m}$ in front of a 47" LCD screen. They each wore a cap with reflective markers and held a joystick with their dominant hand or with their unaffected (non-paretic) hand in the case of hemiparesis. The joystick was fixed to the surface of a table, which was placed in front of the participants (Figure 2). A neuropsychologist conducted all the sessions of the study.

(Figure 2 about here)

Each session consisted of an initial training session during which the participants became acclimated to the interaction mechanisms and the objectives of the task. Specifically, during the training session, each participant was asked to navigate through the virtual environment in the absence of traffic and any audiovisual distractors. Once each participant had acclimated to the system, the main objective of the task was reiterated and the assessment session was performed. 
The assessment session consisted of two consecutive repetitions of virtual street crossing. In each session, the participants were asked to move from the starting point to a large department store (Figure 1) and then to come back as quickly and safely as possible. The virtual task was considered complete when the participants arrived back at the starting point or after the occurrence of four car collisions. After each accident, the rehabilitative therapist encouraged the participant to carefully check both sides of the road before crossing, and the system automatically restarted the session from the initial starting point without discounting the time already spent in the session. Assessment sessions were administered without verbal or visual cues and without visual distractors. Audiovisual feedback was provided when a car passed nearby (a honk) or when an accident occurred (a screeching brake sound and a warning text).

The performances of both the healthy and stroke group participants were recorded. The outcome measures for analysis included the following: 1) the total time spent completing the task (this measure was only recorded for subjects who succeeded in finishing the walk), 2) the number and direction (left and right) of head turns that the subjects performed to check the traffic conditions, 3) the number of accidents (which ranged from 0-4), and 4) the number of near accidents as indicated by warning honks. After finishing the virtual task each patient completed the Short Feedback Questionnaire (SFQm), a modified version of the Witmer \& Singer questionnaire (Witmer \& Singer, 1998). The SFQm uses a 5-point Likert scale on eight questions to measure of the subjective feelings experienced by the participants during the VR session. The SFQm assesses 1) the feeling of enjoyment, 2) the sense of being in the environment, 3) self-awareness of success, 4) the perception of movement control during the scenario, 5) the perception of the environment as realistic, 6) the comprehension of computer feedback, 7) the level of comfort during the experience, and 8) the perception of difficulty while performing the task. The resulting score ranges from 8 to 40 with higher scores indicating a more pleasant subjective experience.

In addition to the VR outcomes and SFQm, the stroke participants were assessed with a 
battery of neuropsychological tests by an expert neuropsychologist who was blinded to the results of the VR task. The neuropsychological battery included standardized tests to measure not only USN but also other attentional and executive cognitive processes (Table 3). It was administered in the 3 days prior to or following the VR session. The outcomes of the neuropsychological tests were compared with the results of the VR session.

(Table 3 about here)

\section{Data analysis}

A comparative analysis of demographic and clinical data of the participants was performed using t-tests and one-way ANOVA with Bonferroni as post-hoc analysis for continuous variables and chi-square or Fisher's exact probability tests for categorical variables. The correlation between the conventional neuropsychological tests and the outcomes of the VR system was analyzed using the Spearman rank correlation coefficient. To avoid the possible bias due to the significant differences detected in the time under treatment between the USN and no-USN group we performed two analyses, including an analysis of covariance (ANCOVA) and a matched-sample analysis. Statistical significance level was defined as $0.05(p<.05)$.

\section{Results}

\section{Feasibility}

All of the control subjects finished the VR session task, while only 17 subjects belonging to the experimental group, 14 participants without USN (93.3\%) and 3 participants with USN (17.6\%), succeeded (Table 4) (Figure 3). All the participants that failed exceeded the maximum number of accidents $(\chi 2=3.1 ; p<.01)$. The participants from the control group completed the task more quickly and safely than the participants from the experimental group, and in turn, the 
participants from the experimental group without USN finished the task more quickly and safely than the participants with USN (time to complete: $F=28.9, p<.01$; number of accidents: $F=55.8$, $p<.01)$. The participants from the experimental group without USN looked to the left $(F=5.6, p<$ $.01)$ and to the right side $(F=3.0, p<.05)$ of the road more often than participants with USN or control subjects. For stroke participants without USN, the number of left turns $(50.9 \pm 47.5)$ was higher than the number of right turns $(32.6 \pm 36.6)$. Since time under treatment was higher in the non-USN group two more in-depth analyses were performed.

First, the analysis of covariance (ANCOVA) with the time under treatment as covariate showed that the interaction between the time under treatment and the presence of neglect was not significant in the custom model $(F=1.6, p=0.2)$. The test also showed that the differences in number of accidents between the USN and the non-USN group persisted in the full factorial model even when controlled for time under treatment $(F=42.5 ; p<.001)$. Furthermore, a lineal regression analysis including the number of accidents as dependent variable, and the presence of neglect, time under treatment, and the interaction between them as independent variables, showed that only the presence of neglect was a significant predictor of the number of accidents $(T=6.5 ; p$ $<.001)$.

Second, since the USN group had higher values of time under treatment and showed a high number of head turns, a sample of participants from the USN ( $n=9)$ and the non-USN group ( $n=9$ ) who were matched (same \pm 2$)$ in terms of head turns $(52.4 \pm 55.4$ vs. $51.5 \pm 55.4$, respectively) were selected. Differences between the non-USN and USN samples persisted: the non-USN sample suffered significantly fewer accidents $(1.2 \pm 0.9$ vs. $3.22 \pm 1.2$, respectively; $p=.001)$ and was significantly more capable of completing the task ( $100 \%$ compared to $30 \%$, respectively; $p=.003)$.

(Table 4 about here) 


\section{Convergent validity}

Correlations were found between the neuropsychological scales and both the time to complete the task and the number of accidents (Table 5).

The time to complete the task (computed for the 17 participants who succeeded) significantly correlated with all timed tests (CPT-Hit RT, CPT-Hit RT Block Change, CTT-TA, CTT-TB, BADS-ZMT1 and BADS-ZMT2) and with the number of errors on the CPT and the raw scores on the BADS tests. The number of head turns to the left and right significantly correlated with the ST-I, and the number of left head turns also correlated with the BIT-S. The number of accidents correlated with the BIT-S, the CTT tests, the raw scores of the BADS tests, and the CPT-Hit RT and number of errors. The number of warning signs slightly correlated with the CPT-Hit RT Block Change.

(Table 5 about here)

\section{Usability}

The global SFQm score was $32.3 \pm 5.8$. The stroke participants $(n=32)$ enjoy the VR experience $(3.8 \pm 1.2)$ and felt being in the environment $(4.1 \pm 1.1)$. They perceived to have succeeded in the task $(3.6 \pm 1.2)$ and to have taken control over it $(3.6 \pm 1.2)$. The participants perceived the environment as being realistic $(4.3 \pm 1.1)$ and understood the computer feedback $(4.2$ $\pm 0.9)$. In general, they did not experience discomfort $(4.5 \pm 0.9)$ or great difficulties while performing the task $(4.2 \pm 1)$. None of the participants felt any side effects during their performance in the virtual environment.

\section{Discussion}

This study presents the feasibility, convergent validity and usability of a new low-cost VR 
street-crossing environment. The performances of healthy controls and stroke participants with and without neglect within the virtual world were compared. These results were also recorded and compared with the results from neuropsychological testing. In addition, subjective information was gathered by questionnaire. Our data confirm the utility of using VEs that simulate real-life activities in stroke rehabilitation. Stroke patients had more difficulty safely crossing the street than did healthy controls. Patients with neglect demonstrated a dramatic lack of efficacy in this task. Our data also suggest that the VR system is potentially both entertaining and motivating, as reflected by the scores from the subjective questionnaire.

The results of the VR session demonstrate that the time to complete the task and the number of accidents were significantly worse in those participants with higher attentional impairments. That is, stroke subjects with USN achieved poorer results (higher values) than subjects without USN, and stroke subjects as a whole achieved poorer results than healthy subjects. These VR outcomes are consistent with the severity of the attentional impairments and support the results of previous studies (Kim et al., 2010; Kim et al., 2004; Peskine et al., 2011; Jannink, Aznar, Kort, van de Vis, Veltink, \& van der Kooij, 2009). Previous research has also demonstrated that head rotations and other parameters can distinguish stroke subjects with USN from those without neglect. For instance, the rotation angle of the head to the left has been reported to be smaller than the rotation angle to the right (Myers \& Bierig, 2000), and the left-to-right reaction time has been reported to be higher (Peskine et al., 2011) in stroke subjects with neglect. In our study, healthy subjects and stroke participants with USN showed a similar and balanced number of head turns to both sides. In the case of healthy controls, this can be understood as the usual environmental search pattern, but in the case of neglect patients, it may reflect a lack of insight into their limitations, which can preclude them from using compensatory strategies. In fact, while this pattern was efficacious for healthy subjects, the same pattern led the stroke participants with USN to suffer more virtual traffic collisions. In addition, the number of head turns was significantly higher in the 
stroke participants without USN than in the other participants. Specifically, the reduced number of head turns to the left shown by subjects with USN in comparison with subjects without USN could be explained by several reasons. First, subjects with USN had shorter time under treatment, which could have limited the acquisition of compensatory strategies that are trained during the rehabilitation period. Second, even having had enough time to learn these strategies, subjects with USN might not have been able to properly use the trained skills when immersed in an ecological environment. Finally, subjects with USN could have tended to minimize the risks associated to a dangerous task such as street-crossing and consequently could have not performed an appropriate scanning because of their lack of awareness. The first of these reasons is merely related to problems in treatment generalization due to temporal constrains. The other two reasons are mainly related to a lack of self-awareness of the deficits and the consequent underestimation of the functional impact derived from those deficits. Our results support this last hypothesis: a covariate analysis showed that the number of head turns was not affected by the time under treatment; also, since the number of head turns could be interpreted as a measure of compensation, a further analysis where subjects were matched in terms of number of head turns showed worse performance of the subjects with neglect. These data suggest that stroke participants with USN made fewer head turns mainly because of their lack of awareness and support the use of the system as a neglect-related activity in an ecological environment. To conclude, the number of near accident warnings did not differentiate between the groups. This indicates that the different groups committed a similar number of risky behaviors. While the stroke participants may have committed risky behaviors unconsciously, the healthy subjects may have been over-confident and taken more conscious risks. These results indicate that participants with different attentional patterns of ability achieved significantly different results in the VR system, demonstrating consistency between the virtual system and the real world.

The results of the correlations between the VR outcomes and the neuropsychological tests 
show that the outcomes of the VR sessions are closely related to measures of vigilance, inattention, impulsivity, sequencing, mental flexibility and planning. The time to complete the task correlated with the timed scales of the neuropsychological battery and with the raw scores of the BADS tests, suggesting an association between time to completion and variables measuring deficits in monitoring, sustained and selective attention, planning ability and planning efficiency. The number of left and right head turns correlated with the interference section of the ST, suggesting that some of these responses could be related to deficits in cognitive inhibition while maintaining the route in the face of traffic intrusions. This could also explain the perseverative use of appropriate scanning strategies in some non-neglected patients, shown by the positive correlation between the raw score of the conventional test of the BIT and the number of head turns to the left. Safe navigation through the virtual environment, as measured in terms of the number of accidents, was associated with measures of attention and executive functioning, cognitive functions usually used in real street crossing. While the CPT correlations suggest that the probability of suffering an accident was related to inattention, reaction time, and impulsivity, the number of near accidents as measured by warning honks depended on vigilance over time. However, some methodological issues, such as ending the exercises after four accidents, could also have influenced these results.

From a technological point of view, our system differs from those previously reported. The system presented by Katz et al. (Katz, Ring, Naveh, Kizony, Feintuch, \& Weiss, 2005) and Weiss et al. (Weiss, Naveh, \& Katz, 2003) had graphical limitations and used the arrow keys of a keyboard to point the avatar's head left, right or forward to see whether vehicles were approaching from either direction. To overcome these limitations, Kim et al. (Kim et al., 2010) and Peskine et al. (Peskine et al., 2011) developed a 3D immersive world and used an HMD in an attempt to provide participants with a more realistic street-crossing scenario. However, the benefits of the increased realism may have been to the detriment of usability and cost. The high SFQm average global score generated by our participants and the individual analysis of each item reinforce the 
usability of our system. The mean scores of the SFQm were above 3 (in a 5-point Likert scale) for all eight items of the questionnaire and were above 4 for five items (presence, realism, feedback, comfort, and ease of use). Specifically, the score for the item related to self-perception of success can be considered as high in absolute terms $(3.6 \pm 1.2)$ but had the lowest scores of the usability questionnaire. The same results were obtained for the item that asked the participants if they had had control over the task. Many participants showed difficulties with the use of the joystick, since it was the first time (the second time, indeed) that they had used it. This two items could have motivated lower values for the item of enjoyment of the VR experience (3.8 \pm 1.2$)$, though this score is also high in absolute terms. In addition to the high scores of the questionnaire, no participants experienced any side effects during their interaction with the virtual system. These considerations demonstrate the ease of using the VR system with stroke patients and argue for its inclusion in neurorehabilitation units.

Some methodological considerations should be noted when analyzing our results. First, although 17 of the 25 patients with right hemispheric stroke were considered to have neglect according to their BIT scores, all showed functional deficits in real-life situations that were observed by their families and by experienced therapists. The use of the conventional tests of the BIT as a cutoff to define USN in our study may partially explain this finding because most paper-and-pencil tests traditionally used to assess USN have been criticized on the basis of lack of ecological validity (Buxbaum et al., 2004). Second, all patients were included in a multidisciplinary program that included rehabilitation techniques to compensate for their deficits. Consequently, the generalization of our results to other populations should be interpreted cautiously. Finally, our sample was not assessed in a real environment; however, the effectiveness of VR for teaching pedestrian safety and the transfer of this knowledge to real world behavior has been previously demonstrated in both healthy subjects (McComas, MacKay, \& Pivik, 2002) and brain injured populations (Katz, Ring, Naveh, Kizony, Feintuch, \& Weiss, 2005). 
However, the results of the present study involving healthy controls and stroke patients with and without USN confirm the clinical effectiveness of the street-crossing system, as shown by the VR outcomes, the scores of the neuropsychological tests, and the correlations between them. In addition, although the system is immersive and realistic, it does not require complex hardware configuration or expensive components, facilitating its integration and use in the clinical setting.

\section{Conclusions}

This paper describes the design and validation of a low-cost VR system to safely train street-crossing in stroke patients. The system allows therapists to conduct challenging sessions under potentially hazardous conditions while allowing the patients to explore repeatedly in a safe environment. The system showed good correlations with measures of attention and executive functions. Although it does not use especially immersive technology, the sense of presence perceived by the participants was high, with the added advantage of its low cost and excellent usability, which enables the integration of the system in a neurorehabilitation service. 


\section{References}

Alderman, N., Burgess, P., Emslie, H., Evans, J. S., \& Wilson, B. (2003). Behavioural assessment of the dysexecutive syndrome (BADS). Journal of Occupational Psychology, Employment and Disability, 5, 33-37.

Allegri, R. F. (2000). Attention and neglect: neurological basis, assessment and disorders. Revista de Neurología, 30, 491-494.

Ansuini, C., Pierno, A. C., Lusher, D., \& Castiello, U. (2006). Virtual reality applications for the remapping of space in neglect patients. Restorative Neurology and Neuroscience, 24, 431-441.

Appelros, P., Karlsson, G. M., Seiger, A., \& Nydevik, I. (2002). Neglect and anosognosia after first-ever stroke: incidence and relationship to disability. Journal of Rehabilitation Medicine, 34(5), 215-220.

Baheux, K., Yoshizawa, M., \& Yoshida, Y. (2007). Simulating hemispatial neglect with virtual reality. Journal of Neuroengineering and Rehabilitation, 4, 27.

Boian, R. F., Burdea, G. C., Deutsch, J. E., \& Winter, S. H. (2004). Street crossing using a virtual environment mobility simulator. Paper presented at 3rd Annual International Workshop on Virtual Reality, Lausanne, Switzerland.

Bowen, A., \& Lincoln, N. B. (2007). Cognitive rehabilitation for spatial neglect following stroke. Cochrane Database Systematic Review, CD003586.

Broeren, J., Samuelsson, H., Stibrant-Sunnerhagen, K., Blomstrand, C., \& Rydmark, M. (2007). Neglect assessment as an application of virtual reality. Acta Neurologica Scandinava, $116,157-163$.

Buxbaum, L. J., Ferraro, M. K., Veramonti, T., Farne, A., Whyte, J., Ladavas, E., et al. (2004). Hemispatial neglect: Subtypes, neuroanatomy, and disability. Neurology, 62, 749-756.

Buxbaum, L. J., Palermo, M. A., Mastrogiovanni, D., Read, M. S., Rosenberg-Pitonyak, E., 
Rizzo, A. A., et al. (2008). Assessment of spatial attention and neglect with a virtual wheelchair navigation task. Journal of Clinical and Experimental Neuropsychology, 30, 650-660.

Castiello, U., Lusher, D., Burton, C., Glover, S., \& Disler, P. (2004). Improving left hemispatial neglect using virtual reality. Neurology, 62, 1958-1962.

Conners, C. K., Epstein, J. N., Angold, A., \& Klaric, J. (2003). Continuous performance test performance in a normative epidemiological sample. Journal of Abnormal Child Psychology, 31, 555-562.

D`Elia, L. F., Satz, P., Uchiyama, C. L., \& White, T. (1996). Color Trails Test: Odessa, FL: PAR.

Folstein, M. F., Folstein, S. E., \& McHugh, P. R. (1975). "Mini-mental state". A practical method for grading the cognitive state of patients for the clinician. Journal of Psychiatry Research, 12, 189-198.

Fordell, H., Bodin, K., Bucht, G., \& Malm, J. (2011). A virtual reality test battery for assessment and screening of spatial neglect. Acta Neurologica Scandinava, 123, 167-174.

Golden, C. J. (1978). Stroop Color and Word Test: A manual for clinical and experimental uses. Chicago, IL: Stoeling Co.

Google. Street View. (2012). Retrieved July 19, 2012, from http://www.google.com/intl/en_ALL/help/maps/streetview/.

Gupta, V., Knott, B. A., Kodgi, S., \& Lathan, C. E. (2000). Using the "VREye" system for the assessment of unilateral visual neglect: two case reports. Presence, 9, 268-286.

Hartman-Maeir A, Katz N. (1995). Validity of the Behavioral Inattention Test (BIT): relationships with functional tasks. American Journal of Occupational Therapy, 49, $507-516$. 
Jannink, M. J., Aznar, M., de Kort, A. C., van de Vis, W., Veltink, P., \& van der Kooij, H. (2009). Assessment of visuospatial neglect in stroke patients using virtual reality: a pilot study. International Journal of Rehabilitation Research, 32, 280-286.

Jehkonen, M., Laihosalo, M., \& Kettunen, J. (2006). Anosognosia after stroke: assessment, occurrence, subtypes and impact on functional outcome reviewed. Acta Neurologica Scandinava, 114, 293-306.

Katz, N., Ring, H., Naveh, Y., Kizony, R., Feintuch, U., \& Weiss, P. L. (2005). Interactive virtual environment training for safe street crossing of right hemisphere stroke patients with unilateral spatial neglect. Disability Rehabilitation, 27, 1235-1243.

Kim, D. Y., Ku, J., Chang, W. H., Park, T. H., Lim, J. Y., Han, K., et al. (2010). Assessment of post-stroke extrapersonal neglect using a three-dimensional immersive virtual street crossing program. Acta Neurologica Scandinava, 121, 171-177.

Kim, J., Kim, K., Kim, D. Y., Chang, W. H., Park, C. I., Ohn, S. H., et al. (2007). Virtual environment training system for rehabilitation of stroke patients with unilateral neglect: crossing the virtual street. Cyberpsychology and Behavior, 10, 7-15.

Kim, K., Kim, J., Ku, J., Kim, D. Y., Chang, W. H., Shin, D. I., et al. (2004). A virtual reality assessment and training system for unilateral neglect. Cyberpsychology and Behavior, 7, 742-749.

Kim, Y. M., Chun, M. H., Yun, G. J., Song, Y. J., \& Young, H. E. (2011). The effect of virtual reality training on unilateral spatial neglect in stroke patients. Annals of Rehabilitation Medicine, 35, 309-315.

Krakauer, J. W. (2006). Motor learning: its relevance to stroke recovery and neurorehabilitation. Current Opinion in Neurology, 19, 84-90.

McComas, J., MacKay, M., \& Pivik, J. (2002). Effectiveness of virtual reality for teaching pedestrian safety. Cyberpsychology and Behavior, 5, 185-190. 
Myers, R. L., \& Bierig, T. A. (2000). Virtual reality and left hemineglect: a technology for assessment and therapy. Cyberpsychology and Behavior, 3, 465-468.

NaturalPoint. TrackIR 4:PRO. (2012). Retrieved July 19, 2012, from http://www.naturalpoint.com/trackir/02-products/product-TrackIR-4-PRO.html.

Peskine, A., Rosso, C., Box, N., Galland, A., Caron, E., Rautureau, G., et al. (2011). Virtual reality assessment for visuospatial neglect: importance of a dynamic task. Journal of Neurology Neurosurgery and Psychiatry, 82, 1407-1409.

Rizzo, A. A., \& Buckwalter, J. G. (1997). Virtual reality and cognitive assessment and rehabilitation: the state of the art. Studies in Health Technology and Informatics, 44, 123-145.

Rizzo, A. A., Cohen, I., Weiss, P. L., Kim, J. G., Yeh, S. C., Zali, B., et al. (2004). Design and development of virtual reality based perceptual-motor rehabilitation scenarios. Paper presented at IEEE Engineering in Medicine \& Biology Society.

Romero, M., Sanchez, A., Marin, C., Navarro, M. D., Ferri, J., \& Noe, E. (2012). Clinical usefulness of the Spanish version of the Mississippi Aphasia Screening Test (MASTsp): validation in stroke patients. Neurología, 27, 216-224.

Rose, F. D., Brooks, B. M., \& Rizzo, A. A. (2005). Virtual reality in brain damage rehabilitation: review. Cyberpsychology and Behavior, 8, 241-262.

Schwebel, D. C., \& McClure, L. A. (2010). Using virtual reality to train children in safe street-crossing skills. Injury Prevention, 16, 1-5.

Simpson, G., Johnston, L., \& Richardson, M. (2003). An investigation of road crossing in a virtual environment. Accident Analysis and Prevention, 35, 787-796.

Smith, J., Hebert, D., \& Reid, D. (2007). Exploring the effects of virtual reality on unilateral neglect caused by stroke: four case studies. Technology and disability, 19, 29-40.

Sugarman, H., Weisel-Eichler, A., Burstin, A., \& Brown, R. (2011). Use of novel virtual reality 
system for the assessment and treatment of unilateral spatial neglect: a feasibility study. Paper presented at International conference on virtual rehabilitation, Zürich.

Tanaka, T., Sugihara, S., Nara, H., Ino, S., \& Ifukube, T. (2005). A preliminary study of clinical assessment of left unilateral spatial neglect using a head mounted display system (HMD) in rehabilitation engineering technology. Journal Neuroengineering and Rehabilitation, 2,31 .

Thomson, J. A., Tolmie, A. K., Foot, H. C., Whelan, K. M., Sarvary, P., \& Morrison, S. (2005). Influence of virtual reality training on the roadside crossing judgments of child pedestrians. Journal of Experimental Psychology: Applied, 11, 175-186.

Weiss, P. L., Naveh, Y., \& Katz, N. (2003). Design and testing of a virtual environment to train stroke patients with unilateral spatial neglect to cross a street safely. Occupational Therapy International, 10, 39-55.

Wilson, B., Cockburn, J., \& Halligan, P. (1987). Development of a behavioral test of visuospatial neglect. Archives of Physical Medicine and Rehabilitation, 68, 98-102.

Witmer, B., \& Singer, M. (1998). Measuring presence in virtual environments: a presence questionnaire. Presence: Teleoperators \& Virtual Enviorements, 7, 225-240.

Wu, H., Ashmead, D. H., \& Bodenheimer, B. (2009). Using immersive virtual reality to evaluate pedestrian street crossing decisions at a roundabout. Paper presented at 6th Symposium on appied perception in Graphics and Visualization. Chania. 
Table 1 Review of the VR use in the assessment and rehabilitation of USN

Assessment

Authors

Hardware

Task

Participants

Conclusions

Myers et al. (Myers \& HMD

To interact $\quad 5$ stroke

The rotation

Bierig, 2000)

Head tracke

with objects in

subjects with

angle of the

3 virtual rooms USN

head to the left

was smaller

than to the right

Gupta et al. (Gupta, HMD

To identify and 2 stroke

The eye

Knott, Kodgi, \&

Eye tracker

count objects

subjects with

trajectories

Lathan, 2000)

To tell the time USN

could

4 healthy

distinguish

subjects

between healthy

and stroke

subjects.

Kim et al. (Kim et al., HMD

To gaze at

$12 \mathrm{ABI}$

USN subjects

2004)

Head tracker

virtual balls

subjects with

spent more time

moving

USN

on scanning the

randomly

40 healthy

environment,

subjects (20 made more

with computer errors, and

experience and needed more

20 without cues. 
experience)

Tanaka et al. (Tanaka, HMD

To perform 8 stroke

VR could help

Sugihara, Nara, Ino,

\& Ifukube, 2005)

Video camera

line and star

subjects with

to clarify the

cancellation

USN

neglect area

tests

Baheux et al.

Haptic device

To bisect lines

2 stroke

No conclusive

(Baheux, Yoshizawa, 3D video

\& Yoshida, 2007)

display

Eye tracker

3D glasses

To press on the

Haptic device

targets while

subjects (4

ignoring the

subjects with

distractors

USN and 4

subjects

recovered from

USN)

Buxbaum et al.

Video display

To name

9 stroke

Correlations

(Buxbaum et al.,

Wheelchair

objects

subjects with

among real and

2008) encountered

along a

pathway 
Jannink et al.

(Jannink, Aznar, Kort, Head tracker

van de Vis, Veltink,

\& van der Kooij,

2009)

2010)
HMD

Head tracker

Head tracker

\section{To detect}

moving balls

to face them

and to press a

button.

To press a

button while

facing a car

that appears in

the virtual

environment
12 stroke

subjects (6

subacute and 6

chronic

subjects)

6 healthy

subjects

Stroke subjects

spent more time

on the task and

had greater

reaction time

time.

Subacute

patients showed

much more

fuzzy search

patterns.

16 stroke

USN subjects

subjects with had greater

USN

deviation angles

and left-to-right

reaction time,

and needed

more cues.

To complete $4 \quad 31$ stroke $\quad$ VR tests

virtualized

subjects $(9$

moderately

tests (baking subjects with correlated with

tray test, line USN and 22 conventional

bisection, star without USN) tests. VR

cancellation,

system 


\begin{tabular}{|c|c|c|c|c|}
\hline & & and visual & & identified the \\
\hline & & extinction) & & USN subjects. \\
\hline Peskine et al (Peskine & HMD & To locate and & 9 stroke & USN subjects \\
\hline et al., 2011) & Head tracker & count some & subjects $(5$ & omitted more \\
\hline & & targets while & with USN) and & targets than \\
\hline & & moving & 9 controls & controls \\
\hline & & forward via & & \\
\hline & & mouse click in & & \\
\hline & & a virtual town & & \\
\hline
\end{tabular}

\section{Rehabilitation}

Authors

Castiello et al.

(Castiello, Lusher,

Burton, Glover, \&

Disler, 2004; Ansuini,

Pierno, Lusher, \&

Castiello, 2006)

Katz et al. (Katz,

Ring, Naveh, Kizony,

Feintuch, \& Weiss,
Hardware Task

Video display

Data glove

reach objects

at incongruent

real and virtual

distances

\section{Participants Conclusions}

6 stroke

subjects with

USN

responses
Increase in the

correct left

Smith et al. (Smith, IREX

There were no

Hebert, \& Reid, 2007)

To reach items 4 stroke

Video display

Keyboard

street

USN

subjects with

USN conclusive

improvement 
Kim et al. (Kim et al., IREX 2007; Kim, Chun,

Yun, Song, \& Young,

Sugarman et al.

(Sugarman,

Weisel-Eichler,

Burstin, \& Brown,
To reach items 24 stroke

subjects with

USN (12

subjects

received

conventional

therapy and 12

subjects

trained with

the IREX)

To reach items 1 stroke

subject with

USN
The IREX

group

significantly

improved in the

scores of star

cancellation test

and the

Catherine

Bergego scale

The balance

between left and

right errors

improved

2011)

The table shows a review of the VR uses on the assessment and training of USN in stroke population from 2000 to current date. In those studies where more than one paper has been published only the most significant paper (according to the design of the study and the relevance of the results) are cited. 
Table 2. Characteristics of the participants

$\begin{array}{lcc}\text { Control group } & \text { Experimental group } & \text { Significance } \\ (n=15) & \text { Non-USN } & \text { USN }(n=17) \\ & \\ & (n=15)\end{array}$

Gender (n, \%)

Male

Female

Age (years)

Education (years)

Chronicity (days)

Time

$$
\text { under }
$$

$12(80 \%)$

$8(47 \%)$

$12(71 \%)$

NS

$3(20 \%)$

$54.6 \pm 5.7$

$50.8 \pm 13.5$

$58.5 \pm 10.1$

NS

treatment (days)

Lesion side (n, \%)

Right

Left

BIT

Participant 1
$11.3 \pm 2.7$
$12.2 \pm 4.3$
$11.5 \pm 4.5$
NS

$482.9 \pm 216.8 \quad 322.6 \pm 243.9$

NS

$457.1 \pm 335.7 \quad 182.4 \pm 44.2 \quad p<.001$ 


\begin{tabular}{|c|c|c|}
\hline Participant 2 & 146 & - \\
\hline Participant 3 & 141 & - \\
\hline Participant 4 & 140 & - \\
\hline Participant 5 & 144 & - \\
\hline Participant 6 & 146 & - \\
\hline Participant 7 & 141 & - \\
\hline Participant 8 & 131 & - \\
\hline Participant 9 & 135 & - \\
\hline Participant 10 & 137 & - \\
\hline Participant 11 & 145 & - \\
\hline Participant 12 & 146 & - \\
\hline Participant 13 & 142 & - \\
\hline Participant 14 & 138 & - \\
\hline Participant 15 & 140 & - \\
\hline Participant 16 & - & 89 \\
\hline Participant 17 & - & 94 \\
\hline Participant 18 & - & 105 \\
\hline Participant 19 & - & 90 \\
\hline
\end{tabular}


Participant 20

Participant 21

Participant 22

Participant 23

Participant 24

Participant 25

Participant 26

Participant 27

Participant 28

Participant 29

Participant 30

Participant 31

Participant 32
105

94

82

128

114

127

108

127

85

90

97

110

47

The table shows the characteristics of the participants. Age, education, chronicity, and mean BIT score are defined in terms of mean and standard deviation. Gender and lesion side are also expressed as a percentage of the number of subjects of each group. Individual BIT scores are also listed. NS: non-significant 
Table 3. Neuropsychological measures

Scale/Test Cognitive domain Outcome measure

Behavioral Inattention

Test (BIT) (Wilson,

Cockburn, \& Halligan,

1987)

Conventional Visual neglect $\quad$ Raw score (BIT-S)

subtest

Conner's Continuous Attention, vigilance $*$ Hit reaction time (CPT-Hit RT)

Performance Test-II and impulsivity

*Hit reaction time block change (CPT-Hit

(Conners, Epstein,

Angold, \& Klaric,

2003)

Number of errors: omissions + commissions (CPT-E)

Stroop test (Golden, Concentration and Interference (ST-I)

inhibition

Color Trail Test

Cognitive

*Time to complete the part A (CTT-TA)

(D’Elia, Satz,

flexibility, mental

*Time to complete the part B (CTT-TB)

Uchiyama, \& White, processing speed

1996)

and visuomotor

skills 
Behavioral Assessment

of the Dysexecutive

Syndrome (Alderman,

Burgess, Emslie,

Evans, \& Wilson,

2003)

Zoo Map Test Planning skills $\quad$ Part 1 raw score: BADS-ZMT-S1

Part 2 raw score: BADS-ZMT-S2

*Time to complete the part 1

(BADS-ZMT-T1)

Key Search Test Problem solving *Time to complete the part 2

(BADS-ZMT-T2)

Raw score (BADS-KST-S)

Description of the tests used in the study and their respective outcomes. More in-depth descriptions can be found in the referenced articles. *Timed outcomes are expressed in seconds. 
Table 4. Outcome measures of the VR session

$\begin{array}{ccc}\text { Control } & \text { Experimental group } & \text { Significance } \\ \operatorname{group}(n=15) & \text { Non-USN } & \text { USN }(n=17) \\ & \\ & (n=15) & \end{array}$

Time to complete $\quad 196.5 \pm 53.1 \quad 556.1 \pm 242.2 \quad 985.3 \pm 303.0 \quad F=28.9 * *\left(a^{* *}\right.$, the task (s)

$\left.\mathrm{b} * *, \mathrm{c}^{* *}\right)$

Head turns (n)

\begin{tabular}{|c|c|c|c|c|}
\hline Right & $16.2 \pm 3.6$ & $32.6 \pm 36.6$ & $13.9 \pm 15.6$ & $F=3^{*}\left(\mathrm{~b}^{*}\right)$ \\
\hline Left & $16.3 \pm 4.1$ & $50.9 \pm 47.5$ & $16.7 \pm 30.7$ & $\begin{array}{c}F=5.6^{* *}\left(\mathrm{a}^{*},\right. \\
\left.\mathrm{b}^{*}\right)\end{array}$ \\
\hline umber of & $0.3 \pm 0.4$ & $1.2 \pm 1.2$ & $3.6 \pm 0.9$ & $F=55.8^{* *}\left(\mathrm{a}^{*}\right.$ \\
\hline cidents (n) & & & & $\left.\mathrm{b}^{* *}, \mathrm{c}^{* *}\right)$ \\
\hline umber of & $10.2 \pm 4.5$ & $12.0 \pm 4.8$ & $11.4 \pm 5.0$ & NS \\
\hline
\end{tabular}

The table shows the results of the VR outcomes. a: control group vs. stroke participants without USN. b: stroke participants without USN vs. stroke participants with USN. c: control group vs. stroke participants with USN.*: $p<.05 . * *: p<.01$. NS: non-significant 
Table 5. Correlations of the neuropsychological tests with the VR outcome measures

$\begin{array}{cccc}\text { Time to Head turns to Head turns to } & \text { Number of } & \text { Number of } \\ \text { complete the the right } & \text { the left } & \text { accidents } & \text { warning } \\ \text { task } & & \end{array}$

BIT-S

$0.4 * \quad-0.7 * *$

CPT-Hit RT

$0.5^{*}$

$0.6^{* *}$

CPT-Hit RT Block

$0.6^{*}$

$-0.3^{*}$

change

CPT-E

$0.6 * *$

$0.5^{* *}$

ST-I

$0.4^{*} \quad 0.5^{* *}$

CTT-TA

$0.75^{* *}$

$0.5^{* *}$

CTT-TB

$0.55^{*}$

$0.6^{* *}$

BADS-KST-S

$-0.5^{* *}$

$-0.4^{*}$

BADS-ZMT-S1

$-0.65 * *$

$-0.5 * *$

BADS-ZMT-S2

$-0.4^{*}$

$-0.3^{*}$

BADS-ZMT-T1 $0.4^{*}$

BADS-ZMT-T2 $0.5^{*}$

Correlation matrix of the VR outcome measures with the psychological tests. Blank values mean non-significant. *: $p<.05, * *: p<.01$. 


\section{Figure Captions}

Figure 1. Virtual scenario.

The virtual street-crossing system provides a first-person view of the environment. The system recreates a real residential area in the city of Valencia (Spain). The figure shows a) real captures of the Google Street View application (Google, 2012), b) snapshots of the virtual street-crossing system, and c) the recreated area of the scenario. The participants can freely move within the highlighted area (free mode) or go from point A to point $\mathrm{B}$, where a large department store is placed (session mode).

Figure 2. Participant interacting with the system.

Participants' interaction was defined by their head rotations, estimated by an infrared camera (a) from the orientation of the constellation of reflective marks (b), and by their displacement in the virtual environment, estimated by the joystick (c).

Figure 3. Graphical representation of the outcome measures of the VR session.

Graphical representation of the time to complete the task (upper left), number of accidents (upper right), head turns to the left (bottom left), and head turns to the right (bottom right) in all the groups. 